\title{
Agent-based Crowd Simulation Considering Emotion Contagion for Emergency Evacuation Problem
}

\author{
Hamed Faroqi ${ }^{\text {a, }}$, Mohammad-Saadi Mesgari ${ }^{\text {a }}$ \\ a Dept. of Geodesey \& Geomatics, Centre of GIS Excellency, K. N. Toosi University of Technology- (faroqi.hamed@gmail.com, \\ mesgari@kntu.ac.ir)
}

KEY WORDS: Evacuation, Agent-based simulation, Emotion Contagion, GIS, Emergency

\begin{abstract}
:
During emergencies, emotions greatly affect human behaviour. For more realistic multi-agent systems in simulations of emergency evacuations, it is important to incorporate emotions and their effects on the agents. In few words, emotional contagion is a process in which a person or group influences the emotions or behavior of another person or group through the conscious or unconscious induction of emotion states and behavioral attitudes. In this study, we simulate an emergency situation in an open square area with three exits considering Adults and Children agents with different behavior. Also, Security agents are considered in order to guide Adults and Children for finding the exits and be calm. Six levels of emotion levels are considered for each agent in different scenarios and situations. The agent-based simulated model initialize with the random scattering of agent populations and then when an alarm occurs, each agent react to the situation based on its and neighbors current circumstances. The main goal of each agent is firstly to find the exit, and then help other agents to find their ways. Numbers of exited agents along with their emotion levels and damaged agents are compared in different scenarios with different initialization in order to evaluate the achieved results of the simulated model. NetLogo 5.2 is used as the multi-agent simulation framework with R language as the developing language.
\end{abstract}

\section{INTRODUCTION}

When a building is designed or when a macro festival is prepared, the best configuration for the evacuation of the building has to be known. A bad configuration can be critical. Crowd evacuations represent an abnormal but potential situation where human lives are in risk to suffer any harm or even, to die. Motivations of evacuations can be floods, fires, terrorism, bomb attack, gas leak, biological agents, earthquakes, etc. The aim of a good configuration is to get the faster and more secure evacuation for all the individuals. In the case of moving human crowds through the space the crowd dynamics determine the behavior of the individuals. Human simulations are not reliable as the human rules used in an emergency evacuation situation are different from a simulation when there is no danger for the individual. Variables as panic, velocities or obstacles determine variance.

In the specific case of crowd evacuations it becomes more complex because the number of individuals has the order of thousands or more. How do we simulate the behavior of thousands of individuals in a crowd evacuation situation with statistical significant results? To solve these problems simulation is needed. Simulation will allow us to predict the behavior of the system and get information without interacting with the real system and be able to learn and take decisions from the output of the simulation. That way the evacuation time is known beforehand and this information can be used to take decisions in case of evacuation. For this we need to create a model with rules extracted from the real system.

During emergencies, emotions greatly affect human behavior. Fear, escalating to panic, may lead to non-rational behavior, disorientation etc. Also, emotions levels, expressions and their effect on behavior differ among individuals, depending on their personality. So, for more realistic multi-agent system simulations of emergency evacuations, it is important to incorporate emotions and their effects on the agents. In few words, emotional contagion is a process in which a person or group influences the emotions or behavior of another person or group through the conscious or unconscious induction of emotion states and behavioral attitudes. Generally, the levels of emotion in an emergency situation would be: Calm, Alarm, Fear, Terror, Panic, and Hysteria. Each of these levels would have different effects on the individual's behavior.

In this study, we simulate an emergency situation in an open square area with three exits considering Adults and Children agents with different behavior. Also, Security agents are considered in order to guide Adults and Children for finding the exits and be calm. Six levels of emotion levels are considered for each agent in different scenarios and situations (a calm agent asks personnel, asks other agents and look around to find its way to exit; however a hysteria agent just move randomly). The simulated agent-based model initialize with the random scattering of agent populations and then when an alarm occurs, each agent react to the situation based on its and neighbors current circumstances. The main goal of each agent is firstly to find the exit, and then help other agents to find their ways. Numbers of exited agents along with their emotion levels and damaged agents are compared in different scenarios with different initialization in order to evaluate the achieved results of the simulated model. NetLogo 5.2 is used as the multi-agent simulation framework with $\mathrm{R}$ language as the developing language.

\section{LITERATURE REVIEW}

There are three main reasons for developing computer simulations for crowd behaviours: firstly, to test scientific

\footnotetext{
${ }^{1}$ Corresponding author
} 
theories and hypotheses; secondly, to test design strategies; and finally, to recreate the phenomena about which to theorize (Pan et al., 2007). Computer models for emergency and evacuation situations have been developed and much research on panics has been of empirical nature and carried out by researchers from the social sciences (Helbing et al., 2001; Reynolds et al., 1987; Coelho et al., 1997; Cordeiro, 2009).

Santos et al. (Santos et al., 2004) offer a critical review of selected simulation models of evacuation. Also, authors have identified social sciences approaches that could improve contemporary simulation models. They argue that social sciences could provide important new directions to simulation models of emergency evacuations. Helbing et al. (Helbing et al., 2001) developed a continuous pedestrian model based on plausible interactions, and pointed out that pedestrian dynamics shows various collective phenomena, such as arching, clogging and herding (Reynolds et al., 1987). According to their findings, every simulation model should reproduce such behaviours in order to be rather realistic. They believe that the above models can serve as an example linking collective behaviour as a phenomenon of mass psychology (from the socio-psychological perspective) to the view of an emergent collective pattern of motion (from the perspective of physics). Their simulations suggest that the optimal behaviour in escape situations is a suitable mixture of individualistic and herding behaviour. Kaup et al. (Kaup et al., 2006) present a simulation model for emergency planning and crowd management purposes. Other simulation studies, combined with optimization algorithms, aim at improving the evacuation efficiency. This can be achieved in terms of evacuation times, assessment and analysis of evacuation plans, as well as routes/path optimizations. Coelho (Coelho, 2010) has deeply studied the mathematical and analytical models, comparing and analyzing them on a systematic basis. In Klüpfel et al. (Klüpfel et al., 2009), the analysis of evacuation processes on-board is taken into consideration. Here authors apply cellular automata to reproduce crowd motion for the detection of possible bottleneck situations during the evacuation process. Filippoupolitis et al. (Filippoupolitis et al., 2008) address the building evacuation optimization problem. Su et al. (Su et al., 2008) developed a discrete-event computer simulation model for assessing evacuation programs and provide a comprehensive idea of evacuation plans for hospital buildings in the event of a possible bomb threat.

\section{METHODOLOGY}

In this section, transition diagrams for each agent (Adult, Child, and security) are depicted and described. Also, emotional levels are defined and explained in details. Moreover, the characteristic of simulated area with the three exits for evacuation is explained.

There would be three kinds of agents with specific emotional levels and decision making process. First kind is Adults agents that have six levels of emotional contagion (Calm $\rightarrow$ Alarm $\rightarrow$ Fear $\rightarrow$ Terror $\rightarrow$ Panic $\rightarrow$ Hysteria). Each agent begins the simulation with the Calm status; and if it does not be success in each tick of simulation, its emotional level goes one level worse. Second kind is Children agents that have two levels of emotional congestions (Calm $\rightarrow$ Hysteria). Each child agent would have two parents that follow them in calm status, or looking for security agents in neighbourhoods. Third kind of agents is Security agents that always are calm and show the direction to the exits. Figure 1 simplify shows the life cycle and decision making process by each agent.

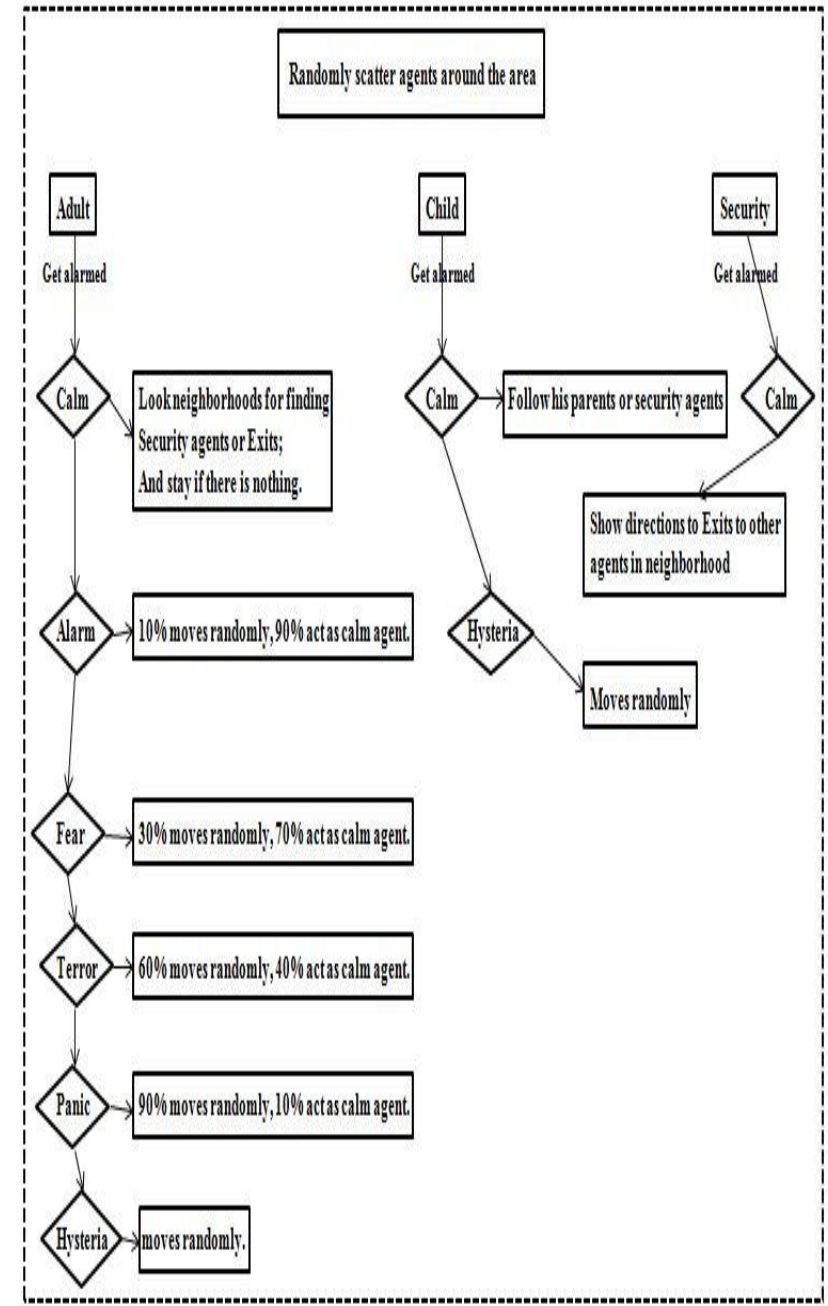

Figure 1. Transition diagrams of agents

The simulated world in NetLogo is an open area with three exits in north, east, and west. The open area is similar to one of most important city halls at Tehran city. Each agents when receive to near the exits would be disappeared from the world. The neighbourhood for each agent is the next two Patch (to the right, left, bottom, up). Figure 2 shows the simulated area. Blue points are Adult agents; yellow points represent children agents; and red point presents security agents.

The simulated world begins its existence in an urgent situation, in which agents want to find the exits to rescue from any harm. The security agents are stable at their initiate places to guide any agent that in their neighbourhood in order to show them the exit direction. So, the security agents would be stay at the emergency situation until the last adult or child agent leave the area, actually find the exits. 


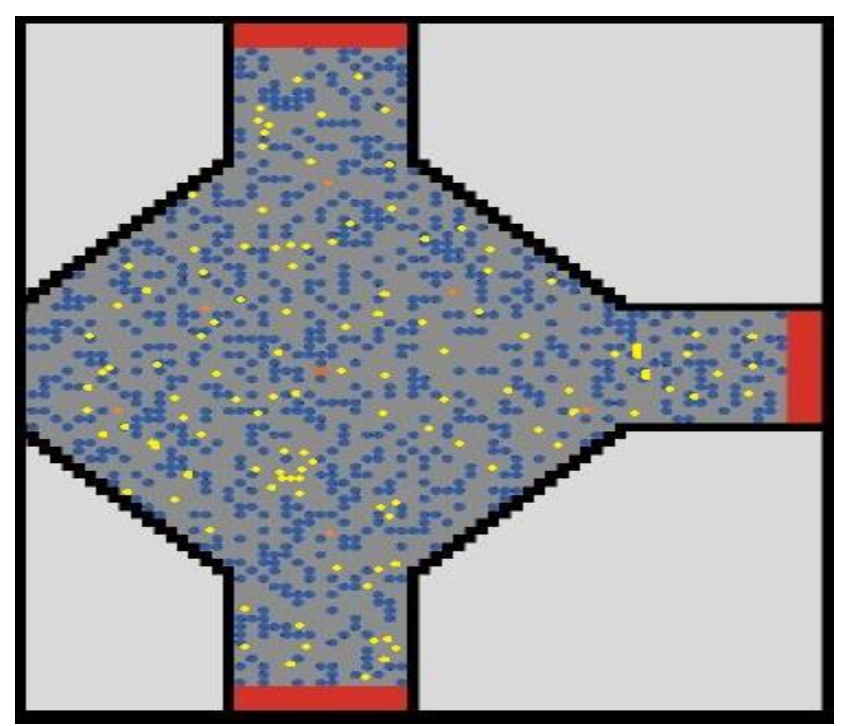

Figure 2. Open area with three exits and agents scattered

\section{IMPLEMENTATION \& RESULTS}

The simulated area and agents would be implemented with different numbers of agents. Initial location of each agent is determined randomly in the area. Four scenarios are implemented. Different rational between counts of three kinds of agents are considered. Table 1 shows the various initialize parameters of agents.

\begin{tabular}{|c|c|c|c|}
\hline No. & Adults & Children & Security \\
\hline 1 & 200 & 40 & 5 \\
\hline 2 & 300 & 50 & 10 \\
\hline 3 & 400 & 60 & 15 \\
\hline 4 & 500 & 70 & 25 \\
\hline
\end{tabular}

Table 1. Initialize counts of agents in scenarios

Four initialized scenarios are run with different counts of agents that are presented above. Figure 3 shows the leaving diagrams for each scenario.

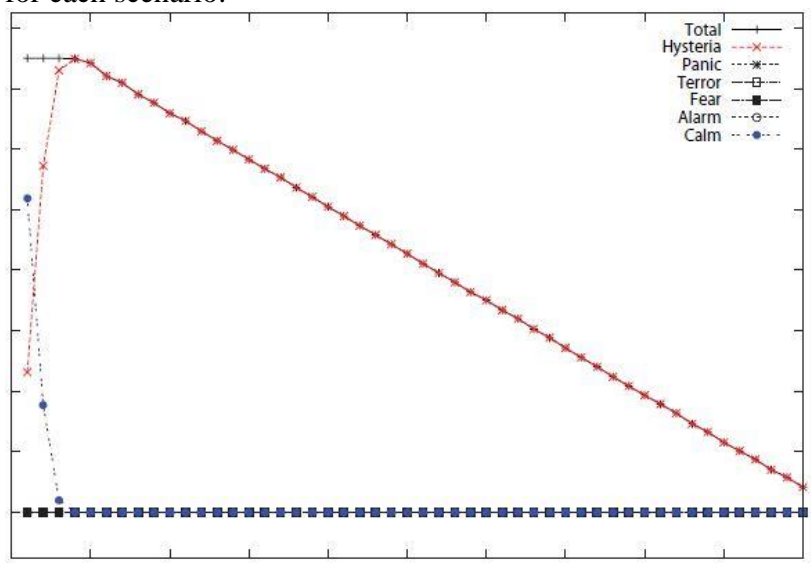

(a)

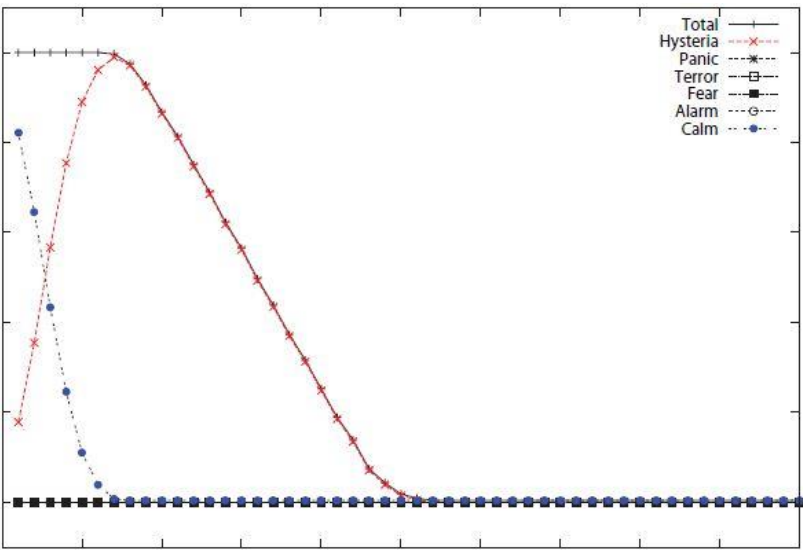

(b)

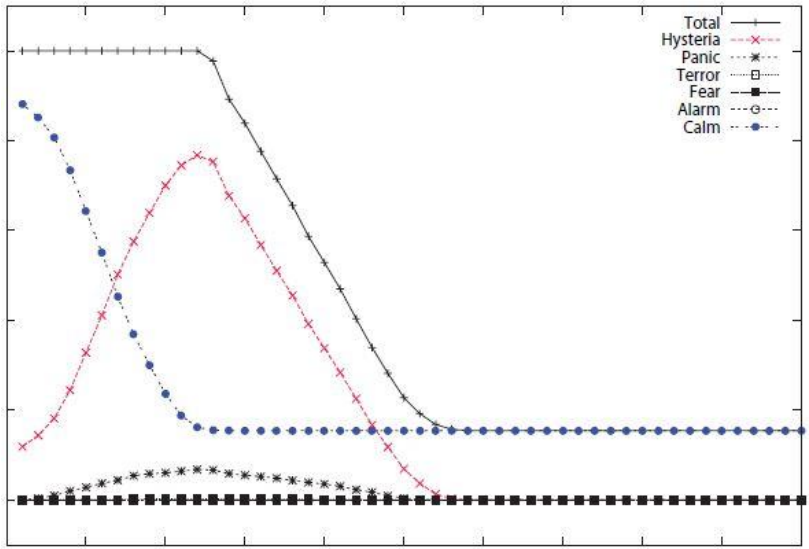

(c)

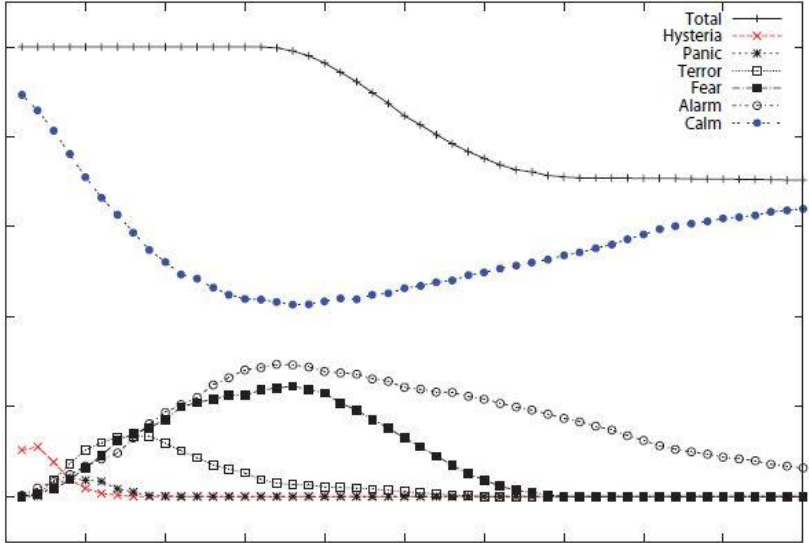

(d)

Figure 3. Agents exiting diagrams: a) scenario 1 ; b) scenario 2; c) scenario 3 ; d) scenario 4

As it was presented in the diagrams, each scenario has its own specific characteristic with different percentage of rescued people with emotional level from calm to hysteria. Scenario 2 has the most number of quitted in the minimum possible time. Scenario 1 has the second amount of exited agents that takes all the simulation time. Scenario 4 has the lowest counts of rescue people; however, most of them in calm status.

It would be possible that the proportion between counts of Adults and Children agents with the numbers of security agents affect the emotional level and percentage of agents that find the 
exits. Table 2 shows the mentioned proportion and the percentage of the rescued agents.

\begin{tabular}{|c|c|c|c|}
\hline $\begin{array}{c}\text { No. of } \\
\text { Scenario }\end{array}$ & $\begin{array}{c}\text { Total numbers of } \\
\text { Agents }\end{array}$ & $\begin{array}{c}\text { Related } \\
\text { Proportion }\end{array}$ & $\begin{array}{c}\text { Rescued } \\
\text { Agents (\%) }\end{array}$ \\
\hline 1 & 240 & $1 / 48$ & $82 \%$ \\
\hline 2 & 350 & $1 / 35$ & $99 \%$ \\
\hline 3 & 460 & $1 / 31$ & $57 \%$ \\
\hline 4 & 570 & $1 / 22$ & $18 \%$ \\
\hline
\end{tabular}

Table 2. Statistics of scenarios

\section{CONCLUSION}

In this study, a multi-agent simulation system is designed and implemented to investigate the urgent evacuation from the square area with the three exits. Also, emotional status is considered for each agent. Agents are divided into three kinds; first group is Adults that have six levels of emotional status; second one is Children that have two levels of emotional status; and the last group is related to security group that have one level of emotional status.

The achieved results and the depicted diagrams show the relation between emotional level, counts of agents, and the proportion between numbers of security agents with the percentage of rescued agents. Regarding results, the proportion of $1 / 35$ is the best one in order to have most rescued people in the emergency situations.

To further studies, it is possible to simulate connected halls with corridors and stairs. Also, it is possible to analyse real video from the evacuations in order to increase our insights for simulating more practical emergency situations. Moreover, the defect of most simulations is the validation that is a difficult process due to the lack of real data.

\section{REFERENCES}

D. Helbing, I. Farkas, P. Molnar and T. Vicsek., 2001. Simulating of pedestrian crowds in normal and evacuation situations, in M.Schreckenberg, S.D. Sharma(ed.) Pedestrian and Evacuation Dynamics. Springer Verlag Berlin and Heidelberg, pp. 21-58.

X. Pan, C.S. Han, K. Dauber and K.H. Law., 2007. A multiagent based framework for the simulation of human and social behaviors during emergency evacuations. AI \& Society.

C. W. Reynolds., 1987. Flocks, Herds, and Schools: A Distributed Behavioral Model. Proceedings of SIGGRAPH '87, Computer Graphics, 21(4), pages 25-34.

A.L Coelho., 1997. Building Evacuation modeling Subject to the action of a fire. Ph.D. Dissertation, LNEC, Lisboa.

E. Cordeiro., 2009, The Influence of Emotions and their limitations in the Building Evacuation, LNEC, Lisboa

D.J. Kaup, T.L. Clarke, L. Malone and R. Olosn., 2006. Crowd Dynamics Simulation Research. Proceedings of the Summer Computer Simulation Conference. Calgary, Canada, pp. 365370 .
A.L Coelho., 2010. Fires in buildings and Emotion Cognation. Edições Orion, Lisboa

Klüpfel, H., Meyer-König, T., Wahle, J., \& Schreckenberg, M., 2001. Microscopic simulation of evacuation processes on passenger ships. In Theory and practical issues on cellular automata (pp. 63-71). Springer London..

A. Filippoupolitis, E. Gelenbe, D. Gianni, L. Hey, G. Loukas and S. Timotheou., 2008. Distributed Agent-based Building Evacuation Simulator. Proceedings of the Summer Computer Simulation Conference. Edinburgh, Scotland, pp. 46- 53.

S. Su, S.T. Tsai, C.L.Shih, R.J.Kuo, H.C.Wang and J.C.Chen., 2008. Use of Computer Simulation in the Evacuation System for Hospitals. Proceedings of the Summer Computer Simulation Conference. Edinburgh, Scotland, pp. 205-212.

G. Santos and B.E. Aguirre., 2004. A Critical Review of Emergency Evacuation Simulation Models. NIST Workshop on Building Occupant Movement during Fire Emergencies June 910. National Institute of Standards and Technology, U.S. Department of Commerce. 